\title{
The effect of human mesenchymal stem cell injection on pain behavior in chronic post-ischemia pain mice
}

\author{
Sie Hyeon Yoo ${ }^{1}$, Sung Hyun Lee ${ }^{2}$, Seunghwan Lee ${ }^{3}$, Jae Hong Park ${ }^{3}$, Seunghyeon Lee ${ }^{4}$, Heecheol Jin ${ }^{4}$, and \\ Hue Jung Park ${ }^{3}$
}

'Department of Anesthesiology and Pain Medicine, Soonchunhyang University Cheonan Hospital, Soonchunhyang University College of Medicine, Cheonan, Korea

${ }^{2}$ Department of Anesthesiology and Pain Medicine, Kangbuk Samsung Hospital, Sungkyunkwan University School of Medicine, Seoul, Korea ${ }^{3}$ Department of Anesthesiology and Pain Medicine, Seoul St. Mary's Hospital, College of Medicine, The Catholic University of Korea, Seoul, Korea ${ }^{4}$ Department of Anesthesiology and Pain Medicine, Soonchunhyang University Bucheon Hospital, Soonchunhyang University College of Medicine, Bucheon, Korea

Received July 15, 2019

Revised October 29, 2019

Accepted November 1, 2019

\section{Correspondence}

Hue Jung Park

Department of Anesthesiology and Pain Medicine, Seoul St. Mary's Hospital, College of Medicine, The Catholic University of Korea, 222 Banpo-daero, Seocho-gu, Seoul 06591, Korea Tel: +82-2-2258-2236

Fax: +82-2-537-1951

E-mail: huejung@catholic.ac.kr
Background: Neuropathic pain (NP) is considered a clinically incurable condition despite various treatment options due to its diverse causes and complicated disease mechanisms. Since the early 2000s, multipotent human mesenchymal stem cells (hMSCs) have been used in the treatment of NP in animal models. However, the effects of hMSC injections have not been studied in chronic post-ischemia pain (CPIP) mice models. Here, we investigated whether intrathecal (IT) and intrapaw (IP) injections of hMSCs can reduce mechanical allodynia in CPIP model mice.

Methods: Seventeen CPIP C57/BL6 mice were selected and randomized into four groups: IT sham $(n=4)$, IT stem $(n=5)$, IP sham $(n=4)$, and IP stem $(n=4)$. Mice in the IT sham and IT stem groups received an injection of $5 \mu \mathrm{L}$ saline and $2 \times 10^{4}$ hMSCs, respectively, while mice in the IP sham and IP stem groups received an injection of $5 \mu \mathrm{L}$ saline and $2 \times 10^{5} \mathrm{hMSCs}$, respectively. Mechanical allodynia was assessed using von Frey filaments from pre-injection to 30 days post-injection. Glial fibrillary acidic protein (GFAP) expression in the spinal cord and dorsal root ganglia were also evaluated.

Results: IT and IP injections of hMSCs improved mechanical allodynia. GFAP expression was decreased on day 25 post-injection compared with the sham group. Injections of hMSCs improved allodynia and GFAP expression was decreased compared with the sham group.

Conclusions: These results suggested that hMSCs may be also another treatment modality in NP model by ischemia-reperfusion.

Key Words: Ganglia, Spinal; Glial Fibrillary Acidic Protein; Hyperalgesia; Mesenchymal Stromal Cells; Mice; Neuralgia; Reperfusion Injury; Spinal Cord; Stem Cells

\section{INTRODUCTION}

Neuropathic pain (NP) is a progressive nervous system disease, occurring in association with a primary lesion or dysfunction in the nervous system [1]. NP is often associated with the presence of abnormal sensory symptoms, such as hyperalgesia, allodynia due to trauma, viral infection (herpes zoster, etc.), metabolic disease, nutritional (c) This is an open-access article distributed under the terms of the Creative Commons Attribution Non-Commercial License (http://creativecommons.org/licenses/by-nc/4.0/), which permits unrestricted non-commercial use, distribution, and reproduction in any medium, provided the original work is properly cited.

(C) The Korean Pain Society, 2020
Author contributions: Sie Hyeon Yoo: Writing/manuscript preparation; Sung Hyun Lee: Investigation; Seunghwan Lee: Investigation; Jae Hong Park: Investigation; Seunghyeon Lee: Investigation; Heecheol Jin: Study conception; Hue Jung Park: Study conception. 
deficiency, immune disease, tumors, and ischemic vascular events. Several pharmacological and non-pharmacological treatment options for NP have been introduced. Despite these efforts, the treatment of NP remains challenging and patients often do not respond to any available treatments [1]. In recent years, stem cell therapy has become a promising approach in molecular medicine with several reports of its neuroprotective effect in various models of nerve injury, suggesting that it may also be useful in the treatment of NP [2-5]. However, the efficacy of stem cell therapy in the treatment of NP can be affected by several variables including the animal model, time of treatment initiation, types of stem cells, routes of administration, administration amount and frequency, and duration of observation following the administration [6-9].

Some of the key considerations in the experimental design of stem cell therapy for NP in animal models include the number of grafted cells, timing (injection point, duration of treatment, and observation), routes of administration, and types of animal models. In this study, we considered the animal model type and administration routes as key variables.

Most previously published studies on the effects of stem cell treatment used spinal nerve ligation or peripheral nerve injury to induce NP [9-13]. However, no reports have been published to date of stem cell injection for the treatment of NP in chronic post-ischemia pain (CPIP). Furthermore, there have been reports of stem cell treatment using intra-brain [14], intraspinal [15], intravenous $[12,13]$, and intrathecal (IT) $[9,11,16,17]$ routes, but no published report has detailed the intrapaw (IP) injection, one of the local injection routes of stem cells for the treatment of NP. Although stem cells are being used in various NP research, the exact mechanism of action remains elusive. In the treatment of NP, the immunomodulatory properties of mesenchymal stem cells (MSCs), such as reducing levels of pro-inflammatory cytokines (such as interleukin 1 beta [IL-lbeta] and IL-17), astrocyte and microglial activation, and stimulating the secretion of anti-inflammatory cytokines and regenerative molecules, appear to be the key mechanism of action rather than its well-known multipotent differentiation ability [18-22].

Therefore, the authors hypothesized that MSC injections can reduce mechanical allodynia in the CPIP mouse model, an animal model of complex regional pain syndrome (CRPS) type 1 , in which an excessive inflammatory response is implicated. To test this hypothesis, pain behavior and glial fibrillary acidic protein (GFAP) expression in the spinal cord and the dorsal root ganglia (DRG) were examined in CPIP model mice following the IT and IP injections of human mesenchymal stem cells (hMSCs).

\section{MATERIALS AND METHODS}

\section{Animals and CPIP model production}

The animal study protocol was approved by the Institutional Animal Care and Use Committee of the College of Medicine, The Catholic University of Korea (2017-0158-01). Male adult C57/BL6 mice (25-30 g) were used in this study and housed in groups of five, allowed free access to food and water under a 12:12-hr light-dark cycle. All animals were maintained to adapt to their environmental condition for 7 days before the experiment.

The CPIP model was induced in mice under general anaesthesia with isoflurane by placing a tight-fitting $\mathrm{O}$ ring (O-Rings West, Seattle, WA) with a 5/64 inch internal diameter around the left ankle for 3 hours, as described by Coderre et al. [23]. The O-rings were removed while the mice were still under general anesthesia, allowing for reperfusion. Mice in the sham group were placed under general anesthesia, but their ankles were loosely rather than tightly surrounded by cut O-ring. Mechanical allodynia was measured, using von Frey filaments (18011 SemmesWeinstein filament; Stoelting Co., Wood Dale, IL), every 2 days before the stem cell injection from 1 to 7 days after reperfusion.

\section{Stem cell transplantation procedures}

hMSCs (Catholic MASTER Cells) were obtained from the Catholic Institute of Cell Therapy (CIC; Seoul, Korea). The Catholic MASTER Cells supplied by CIC were derived from human bone marrow donated by healthy donors after informed consent.

At day 7 after reperfusion, 17 of the CPIP model mice that exhibited mechanical allodynia were selected and randomized into four groups. Animals were divided into an IT sham group ( $\mathrm{n}=4)$, IT stem group $(\mathrm{n}=5)$, IP sham group $(\mathrm{n}=4)$, and IP stem group $(\mathrm{n}=4)$. The Hylden and Wilcox's method modification [24] was used to perform the IT needle placement. The mice were anesthetized with $3 \%$ isoflurane in a chamber and then the lower back was shaved to help visualize the lumbosacral region, and each mouse was placed in a nose cone for continued isoflurane administration during the procedure. A 30-gauge needle attached to a $50 \mu \mathrm{L}$ Hamilton syringe (Hamilton, Reno, $\mathrm{NV}$ ) was inserted between the L5 and L6 vertebrae.

Five microliters of sterile saline was slowly injected over 30 seconds into each mouse of the IT sham group and $5 \mu \mathrm{L}$ $\left(2 \times 10^{4}\right.$ cells $)$ of stem cells were injected into each mouse of the IT stem group. Sterile saline $(5 \mu \mathrm{L})$ was injected into the hind paw of each mouse in the IP sham group and $5 \mu \mathrm{L}$ $\left(2 \times 10^{5}\right.$ cells $)$ of stem cells were injected into each mouse of 
the IP stem group. The number of cells to be injected was determined based on the study by Kim et al. [25]

\section{Evaluation of mechanical allodynia}

Mechanical allodynia was measured every 2 days after the stem cell and saline injection from 1 to 31 days using the von Frey up-down method. To stimulate the plantar surface, a mouse was placed on a wire mesh floor and a transparent $8 \times 8 \times 18 \mathrm{~cm}$ plastic box was placed over the mouse. After the mouse was acclimated to the environment for 30 minutes or more, a force was vertically applied to the mouse's paw for 3 seconds using a von Frey filament so that the filament was bent in the midplantar area, and the avoidance response was evaluated. Seven filaments weighing 2.44-4.31 g were used. The simplified up-down method used by Bonin et al. [26] was used to examine the reflex 4 additional times starting from when the mouse started or stopped showing an avoidance response. A 50\% response threshold was measured based on the reflex patterns and log-values of the von Frey filament tests. Mechanical values for both the paws were measured and averaged by each limb.

\section{Fluorescence immunochemistry}

At 32 days after the stem cell injection, a total of 17 male mice were deeply anesthetized with ketamine and perfused with $0.9 \%$ saline, followed by $4 \%$ paraformaldehyde in $0.1 \mathrm{M}$ phosphate buffer via the cardiovascular perfusion surgery. The ipsilateral L3-L5 DRG and the spinal cord were collected and post-fixed in $4 \%$ paraformaldehyde, then placed overnight in $25 \%$ sucrose at $4^{\circ} \mathrm{C}$. The DRG and spinal cord tissues were sliced transversely into $10 \mu \mathrm{m}$ sections on a cryostat (Microm HM 525; Thermo Fisher Scientific, Waltham, MA). The slices were incubated with mouse anti-GFAP (1:150; MED Millipore, Temecula, CA) overnight at $4^{\circ} \mathrm{C}$. After the sections were washed with buffer, they were incubated with goat anti-mouse immunoglobulin $\mathrm{G}$ conjugated to fluorescein isothiocyanate (1:200; EarthOx, San Francisco, CA) for 1 hour at $37^{\circ} \mathrm{C}$.

At least 2 pictures from each DRG and spinal cord per mouse were obtained using confocal microscopy (Zeiss LSM/510 Upright 2 photon; Carl Zeiss, Oberkochen, Germany) and stored digitally. The relative fluorescence intensity of the immunofluorescent images was calculated by Image Pro Plus ver. 6.0 software (Media Cybernetics Inc., Rockville, MD). GFAP staining was quantified by measuring the total integrated intensity of the pixels divided by the total number of pixels in a standardized area. Staining intensity was examined in laminae I and II of the superficial dorsal horn using a standardized box with
4-6 mice per experimental condition. Only pixels above a preset background threshold were included, and the GFAP data are presented as the total area above the intensity threshold. The raw values were subjected to statistical analyses.

\section{Statistical analysis}

Statistical analyses were performed using GraphPad Prism ver. 5.0 (GraphPad Software Inc., San Diego, CA) and the data are presented as the group mean \pm standard error of the mean. Paw withdrawal thresholds were analyzed with one-way analysis of variance with repeated measures over time, followed by a multiple comparison test (Dunnett's post hoc test) to compare each time point and group.

\section{RESULTS}

\section{Stem cell injections reduced mechanical allodynia}

The pain withdrawal threshold of the left hind paw significantly increased following the hMSC injection on days 26-31 in the IT stem and IP stem groups compared to the IT sham and IP sham groups (Fig. 1A; $P<0.05$ ). The pain withdrawal threshold of the left hind paw significantly increased following the hMSC injection on days 26-31 in the IT stem and IP stem groups compared to the pre-injection days (Fig. 1A; $P<0.05$ ). There was no statistically significant difference in mechanical allodynia between the IT stem and IP stem groups. In the contralateral hindpaw, there was no statistically significant intergroup difference in the pain withdrawal thresholds (Fig. 1B).

\section{Stem cell injections decreased GFAP expression in CPIP model mice}

The IT stem and IP stem groups showed significantly reduced GFAP expression in the DRG and the spinal cord compared to the IT sham and IP sham groups (Fig. 2; $P<$ $0.001)$.

\section{DISCUSSION}

In this CPIP mouse model, IT and IP hMSC injections improved mechanical allodynia and reduced GFAP expression compared with the sham groups.

Since the 2000s, stem cells have been used as the major source of cell-based therapy for several diseases and medical conditions. MSCs have several advantages, such as a high expansion potential, stable genetic phenotype, and 

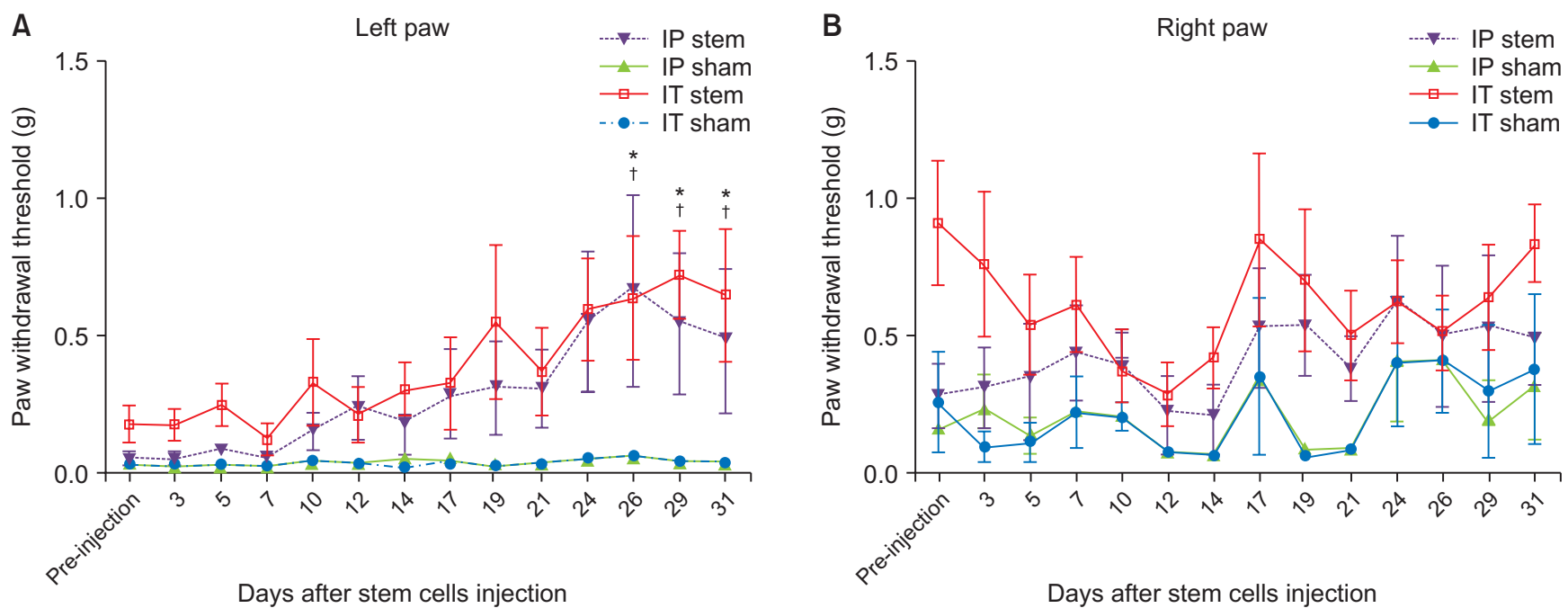

Fig. 1. The anti-allodynic effects of human mesenchymal stem cells (hMSCs) on pain withdrawal threstholds in chronic post-ischemia pain mice model by von Frey filament testing. (A) Paw withdrawal mechanical thresholds were reduced after day 26 in left hindpaw of the intrapaw (IP) stem compared with pre-injection and sham-operated mice groups ( ${ }^{*} P<0.05$ ). Also the pain withdrawal threshold of the left hindpaw significantly increased at day $26-$ 31 after stem cell injection in the intrathecal (IT) stem group compared with pre-injection and sham-operated mice groups $\left({ }^{\dagger} P<0.05\right)$. (B) In the contralateral hindpaw, there no significant difference in pain withdrawal threshold between the groups.

A
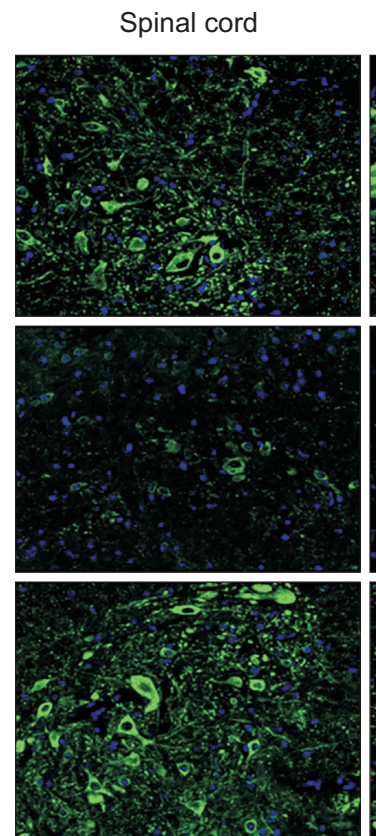

IT sham

IT stem

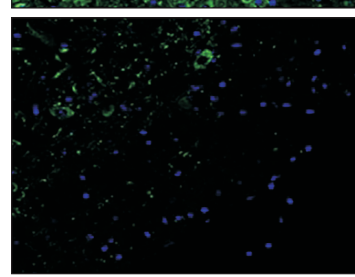

DRG
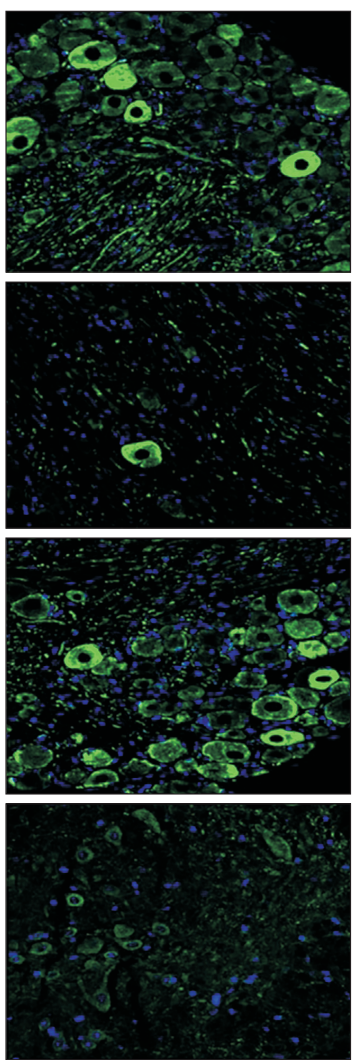

B
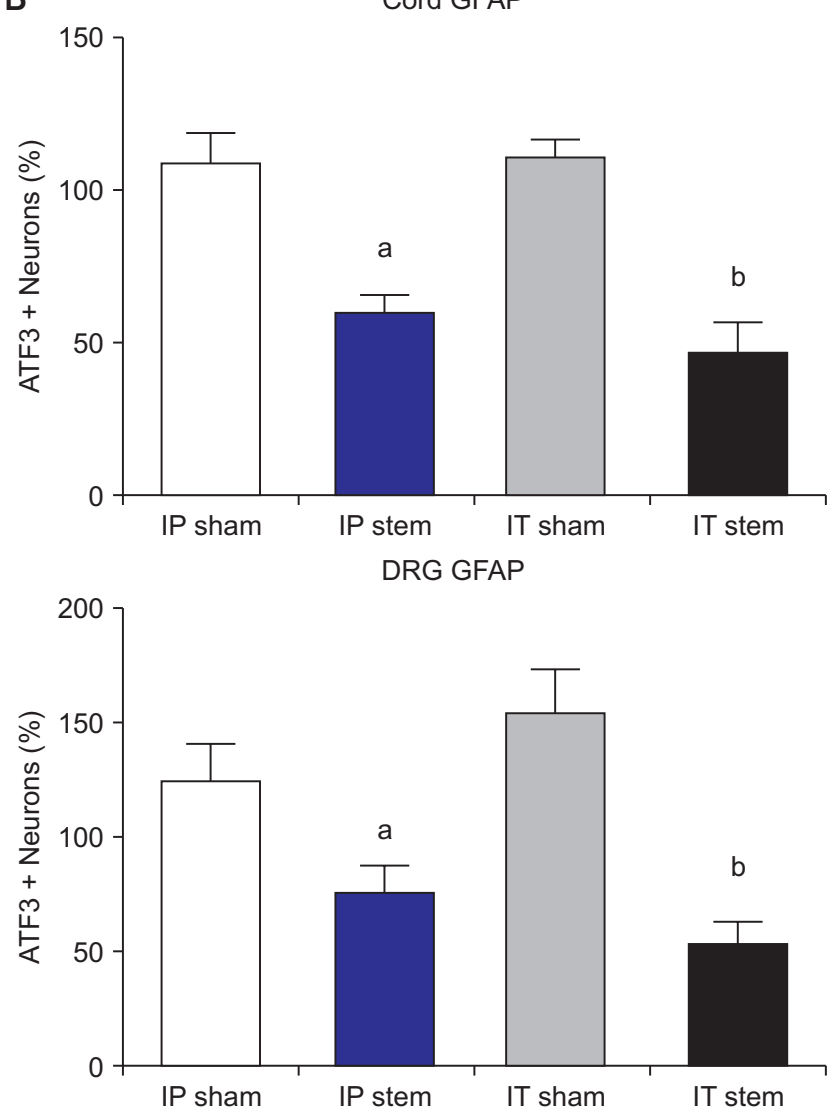

Fig. 2. The expression of glial fibrillary acidic protein (GFAP) in the spinal cord and dorsal root ganglion (DRG). (A) In intrathecal (IT) stem and intrapaw (IP) stem groups, GFAP expression reduced in the spinal cord and DRG (200× magnification). (B) And there is a significant decrease in the staining density of GFAP at day 31 after injection of human mesenchymal stem cells compared with sham groups. ATF3: activating transcription factor $3 .{ }^{a} P<0.001$ vs. IP sham, ${ }^{b} P<0.001$ vs. IT sham. 
strong immunosuppressive properties without immunosuppressant use [16], as well as the potential for autologous and heterologous transplantation [27]. Reports have been published of MSCs in the treatment of NP $[28,29]$, and the administration of stem cells via various routes has been shown to stimulate functional recovery of various brain, spinal cord, and peripheral nerve injuries [10-12,30-32]. However, these studies were conducted using direct nerve injuries in animals, and no report to date has detailed stem cell injections in the treatment of NP in an ischemiareperfusion model, such as the CPIP mice model used in the present study. While the exact mechanism underlying the improvement of pain behavior by MSCs in neuropathic models remains elusive, it is the secretion of neurotrophic molecules and the immunomodulatory properties of MSCs, rather than their multipotent differentiation, which are believed to mediate the recovery of nerve injury by MSCs [33,34].

CRPS-I, formerly known as reflex sympathetic dystrophy, is a clinical syndrome of variable course and unknown cause characterized by pain, swelling, and vasomotor dysfunction of an extremity, as occurring in the absence of definable nerve injury. CRPS-I usually occurs after fracture, sprain, contusion, crush injury, arthroscopic surgery, tight casting, or edematous soft tissue injury [23]. A common characteristic is that these injuries induce an early inflammatory response and microvascular and ischemic changes in tissues. Although the exact pathophysiology of CRPS-I is unknown, a possible mechanism is posttraumatic inflammation [23]. Posttraumatic inflammation is the key mechanism underlying the observed symptoms in the CPIP mouse model, an animal model that exhibits clinical symptoms of CRPS type I $[35,36]$. Therefore, the authors hypothesized that the injection of MSCs with immunomodulatory properties could reduce mechanical allodynia in CPIP model mice.

As we hypothesized, the present study showed that IT and IP injections of hMSCs increased the pain withdrawal threshold on day 31 post-injection in CPIP model mice. One of the key findings of this study is that the onset of the anti-allodynic effect following MSCs in CPIP model mice was slower than that of a direct nerve injury model. Studies using direct nerve injury models showed the antiallodynic effect of MSCs at 3-14 days post-injection; this effect persisted throughout the study $[10-13,16]$. The current study clearly showed a slower onset of the anti-allodynic effect following the MSC injection, measured by the pain withdrawal threshold. To determine whether this difference is relevant, immunohistological evidence compared to the sham group was necessary. In our immunohistologic study, there was a statistically significant reduction in GFAP expression in the spinal cord and DRG in the IT stem and IP stem groups compared to the sham groups, indicating a reduction in the astrocyte reactivity involved in allodynia maintenance. Therefore, the difference in the delayed onset of the anti-allodynic effect may be due to the difference in the experimental animal models.

In addition to the animal models, administration route is a critical consideration in studying the effects of MSCs. The IT administration of MSCs used in the present study was used in many other studies for its various advantages such as being minimally invasive, reaching therapeutic concentrations due to the small IT space, avoiding issues with the immunological barrier (e.g., blood-brain/spinal cord barrier), and placement of injection sites near the injured DRG and spinal cord tissues [16]. Most studies have reported amelioration of mechanical allodynia following the IT administration of MSCs $[11,13,16,17,28]$. However, other reports suggested the IT route, as one reported no improvement in mechanical and heat allodynia following repeated IT administration of $2 \times 10^{6}$ cell/15 $\mu$ g MSCs on days 2-4 after partial sciatic nerve ligation in rats [9]. The relatively short observation period and the animal model used may have affected their results, as our study showed amelioration of mechanical allodynia in MSC-injected groups after day 26 post-hMSCs injection.

The other route of administration, IP, used in this study is considered an intralesional or local administration in nerve injury models. The systemic and local administration of MSCs can reduce inflammation and NP [10,37-39]. However, some studies using spinal cord injury models showed the onset of motor recovery to be 15 days following the intralesional injection of MSCs and 3 months following the intravenous injection. Further, the recovery of cold sensitivity was observed a month following the intralesional injection versus 6 months following the intravenous injection, suggesting that intralesional (local) administration is more effective than systemic administration [15]. In addition, one report detailed improvement in mechanical hyperalgesia and cold allodynia following the local injection of bone marrow mononuclear cells in the hindlimb muscles of diabetic neuropathy rats [40]. In this study, the IT and IP injection groups showed reduced mechanical allodynia compared to the sham groups, suggesting that the IP (local injection) route is also as effective as the IT route.

Although we can't determine the exact reason that the paw withdrawal threshold on the right foot looks more variable compared to those of the left paw, symptoms of CRPS spread to the contralateral side in about $16 \%$ of all patients with CRPS-I [41,42], and there have been reports of symptom contraction in contralateral unlesioned structures following peripheral nerve damage in animal experiments [43]. The contralateral effects are usually mild and brief, and the paw withdrawal threshold on the right foot 
showed more variable compared to that of the left paw.

Despite the positive results of using MSCs in the treatment of NP in many studies, translating the findings to clinical use has been challenging. Some of the limitations of the studies that contribute to this challenge are the lack of a clear understanding of the molecular mechanism in treating chronic pain, the localization and life span of transplanted MSCs, and the ideal condition for MSC injections. The timing of transplantation is another critical consideration. Some studies showed positive results when MSCs were injected at the same time as the nerve injury procedure, but this is neither practical nor achievable in a clinical setting [17]. Most studies transplanted MSCs 3-7 days after the nerve injury [10-13]; therefore, hMSCs were injected 7 days following the induction of CPIP in this study [10-13].

This study has several limitations: (1) This immunohistological study was only performed in the spinal cord and the DRG, and the levels of pro-inflammatory proteins other than GFAP were not measured; (2) The number of animals used in the study was limited due to limitations in the availability of hMSCs; (3) Only a small number of CPIP model mice had persistent mechanical allodynia more than 4 weeks post-reperfusion, making it difficult to observe mechanical allodynia at more than 5 weeks post-reperfusion; and (4) Cold and heat allodynia were not measured.

In summary, the results of this study demonstrate that the IT and IP administration of hMSCs in a non-nerve injury model, the CPIP mouse model, reduced mechanical allodynia, indicating that MSCs may be used in the cell therapy of CRPS type I.

\section{CONFLICT OF INTEREST}

No potential conflict of interest relevant to this article was reported.

\section{FUNDING}

This study was in part supported by the Soonchunhyang University Research Fund.

\section{ORCID}

Sie Hyeon Yoo, https://orcid.org/0000-0002-2629-5367

Sung Hyun Lee, https://orcid.org/0000-0001-5616-6649

Seunghwan Lee, https://orcid.org/0000-0001-9450-5437

Jae Hong Park, https://orcid.org/0000-0003-0973-0985
Seunghyeon Lee, https://orcid.org/0000-0001-5908-3822

Heecheol Jin, https://orcid.org/0000-0003-3231-8294

Hue Jung Park, https://orcid.org/0000-0002-3775-1794

\section{REFERENCES}

1. Hatch MN, Cushing TR, Carlson GD, Chang EY. Neuropathic pain and SCI: identification and treatment strategies in the 21st century. J Neurol Sci 2018; 384: 75-83.

2. Siniscalco D, Rossi F, Maione S. Molecular approaches for neuropathic pain treatment. Curr Med Chem 2007; 14: 17837.

3. Cao Q, Benton RL, Whittemore SR. Stem cell repair of central nervous system injury. J Neurosci Res 2002; 68: 501-10.

4. Lindvall O, Kokaia Z. Stem cell therapy for human brain disorders. Kidney Int 2005; 68: 1937-9.

5. Lindvall O, Kokaia Z. Stem cells for the treatment of neurological disorders. Nature 2006; 441: 1094-6.

6. Klass M, Gavrikov V, Drury D, Stewart B, Hunter S, Denson DD, et al. Intravenous mononuclear marrow cells reverse neuropathic pain from experimental mononeuropathy. Anesth Analg 2007; 104: 944-8.

7. Meirelles Lda S, Nardi NB. Methodology, biology and clinical applications of mesenchymal stem cells. Front Biosci (Landmark Ed) 2009; 14: 4281-98.

8. Amemori T, Jendelová $\mathrm{P}$, Růzicková $\mathrm{K}$, Arboleda $\mathrm{D}$, Syková $\mathrm{E}$. Co-transplantation of olfactory ensheathing glia and mesenchymal stromal cells does not have synergistic effects after spinal cord injury in the rat. Cytotherapy 2010; 12: 212-25.

9. Schäfer S, Berger JV, Deumens R, Goursaud S, Hanisch UK, Hermans E. Influence of intrathecal delivery of bone marrow-derived mesenchymal stem cells on spinal inflammation and pain hypersensitivity in a rat model of peripheral nerve injury. J Neuroinflammation 2014; 11: 157.

10. Siniscalco D, Giordano C, Galderisi U, Luongo L, de Novellis $\mathrm{V}$, Rossi F, et al. Long-lasting effects of human mesenchymal stem cell systemic administration on pain-like behaviors, cellular, and biomolecular modifications in neuropathic mice. Front Integr Neurosci 2011; 5: 79.

11. Zhang EJ, Song CH, Ko YK, Lee WH. Intrathecal administration of mesenchymal stem cells reduces the reactive oxygen species and pain behavior in neuropathic rats. Korean J Pain 2014; 27: 239-45.

12. Franchi S, Valsecchi AE, Borsani E, Procacci P, Ferrari D, Zalfa C, et al. Intravenous neural stem cells abolish nociceptive hypersensitivity and trigger nerve regeneration in experimental neuropathy. Pain 2012; 152: 850-61.

13. Liu L, Hua Z, Shen J, Yin Y, Yang J, Cheng K, et al. Comparative efficacy of multiple variables of mesenchymal stem cell transplantation for the treatment of neuropathic pain in rats. Mil Med 2017; 182: 175-84. 
14. Siniscalco D, Giordano C, Galderisi U, Luongo L, Alessio N, Di Bernardo G, et al. Intra-brain microinjection of human mesenchymal stem cells decreases allodynia in neuropathic mice. Cell Mol Life Sci 2010; 67: 655-69.

15. Vaquero J, Zurita M, Oya S, Santos M. Cell therapy using bone marrow stromal cells in chronic paraplegic rats: systemic or local administration? Neurosci Lett 2006; 398: 12934.

16. Chen G, Park CK, Xie RG, Ji RR. Intrathecal bone marrow stromal cells inhibit neuropathic pain via TGF- $\beta$ secretion. J Clin Invest 2015; 125: 3226-40.

17. Chen C, Chen F, Yao C, Shu S, Feng J, Hu X, et al. Intrathecal injection of human umbilical cord-derived mesenchymal stem cells ameliorates neuropathic pain in rats. Neurochem Res 2016; 41: 3250-60.

18. Bonfield TL, Caplan AI. Adult mesenchymal stem cells: an innovative therapeutic for lung diseases. Discov Med 2010; 9: 337-45.

19. Pan HC, Cheng FC, Chen CJ, Lai SZ, Lee CW, Yang DY, et al. Post-injury regeneration in rat sciatic nerve facilitated by neurotrophic factors secreted by amniotic fluid mesenchymal stem cells. J Clin Neurosci 2007; 14: 1089-98.

20. Kim HK, Park SK, Zhou JL, Taglialatela G, Chung K, Coggeshall RE, Chung JM. Reactive oxygen species (ROS) play an important role in a rat model of neuropathic pain. Pain 2004; 111: 116-24.

21. Pisati F, Bossolasco P, Meregalli M, Cova L, Belicchi M, Gavina $\mathrm{M}$, et al. Induction of neurotrophin expression via human adult mesenchymal stem cells: implication for cell therapy in neurodegenerative diseases. Cell Transplant 2007; 16: 41-55.

22. Siniscalco D, Rossi F, Maione S. Stem cell therapy for neuropathic pain treatment. J Stem Cells Regen Med 2007; 3: 2-11.

23. Coderre TJ, Xanthos DN, Francis L, Bennett GJ. Chronic post-ischemia pain (CPIP): a novel animal model of complex regional pain syndrome-type I (CRPS-I; reflex sympathetic dystrophy) produced by prolonged hindpaw ischemia and reperfusion in the rat. Pain 2004; 112: 94-105.

24. Hylden JL, Wilcox GL. Intrathecal morphine in mice: a new technique. Eur J Pharmacol 1980; 67: 313-6.

25. Kim H, Kim HY, Choi MR, Hwang S, Nam KH, Kim HC, et al. Dose-dependent efficacy of ALS-human mesenchymal stem cells transplantation into cisterna magna in SOD1-G93A ALS mice. Neurosci Lett 2010; 468: 190-4.

26. Bonin RP, Bories C, De Koninck Y. A simplified up-down method (SUDO) for measuring mechanical nociception in rodents using von Frey filaments. Mol Pain 2014; 10: 26.

27. Giordano A, Galderisi U, Marino IR. From the laboratory bench to the patient's bedside: an update on clinical trials with mesenchymal stem cells. J Cell Physiol 2007; 211: 27-35.

28. Lin CR, Wu PC, Shih HC, Cheng JT, Lu CY, Chou AK, et al. In- trathecal spinal progenitor cell transplantation for the treatment of neuropathic pain. Cell Transplant 2002; 11: 17-24.

29. Eaton MJ, Plunkett JA, Martinez MA, Lopez T, Karmally S, Cejas $\mathrm{P}$, et al. Transplants of neuronal cells bioengineered to synthesize GABA alleviate chronic neuropathic pain. Cell Transplant 1999; 8: 87-101.

30. Franchi S, Castelli M, Amodeo G, Niada S, Ferrari D, Vescovi A, et al. Adult stem cell as new advanced therapy for experimental neuropathic pain treatment. Biomed Res Int 2014; 2014: 470983.

31. Siniscalco D, Giordano C, Galderisi U, Luongo L, Alessio N, Di Bernardo G, et al. Human mesenchymal stem cells as novel neuropathic pain tool. J Stem Cells Regen Med 2010; 6: 127.

32. Zurita M, Vaquero J. Functional recovery in chronic paraplegia after bone marrow stromal cells transplantation. Neuroreport 2004; 15: 1105-8.

33. Soleymaninejadian E, Pramanik K, Samadian E. Immunomodulatory properties of mesenchymal stem cells: cytokines and factors. Am J Reprod Immunol 2012; 67: 1-8.

34. Knaän-Shanzer S. Concise review: the immune status of mesenchymal stem cells and its relevance for therapeutic application. Stem Cells 2014; 32: 603-8.

35. Birklein F, Dimova V. Complex regional pain syndrome-upto-date. Pain Rep 2017; 2: e624.

36. Goris RJ. Reflex sympathetic dystrophy: model of a severe regional inflammatory response syndrome. World J Surg 1998; 22: 197-202.

37. Guo W, Wang H, Zou S, Gu M, Watanabe M, Wei F, et al. Bone marrow stromal cells produce long-term pain relief in rat models of persistent pain. Stem Cells 2011; 29: 1294-303.

38. Siniscalco D. Transplantation of human mesenchymal stem cells in the study of neuropathic pain. Methods Mol Biol 2010; 617: 337-45.

39. Musolino PL, Coronel MF, Hökfelt T, Villar MJ. Bone marrow stromal cells induce changes in pain behavior after sciatic nerve constriction. Neurosci Lett 2007; 418: 97-101.

40. Naruse K, Sato J, Funakubo M, Hata M, Nakamura N, Kobayashi $\mathrm{Y}$, et al. Transplantation of bone marrow-derived mononuclear cells improves mechanical hyperalgesia, cold allodynia and nerve function in diabetic neuropathy. PLoS One 2011; 6: e27458.

41. Allen G, Galer BS, Schwartz L. Epidemiology of complex regional pain syndrome: a retrospective chart review of 134 patients. Pain 1999; 80: 539-44.

42. Maleki J, LeBel AA, Bennett GJ, Schwartzman RJ. Patterns of spread in complex regional pain syndrome, type I (reflex sympathetic dystrophy). Pain 2000; 88: 259-66.

43. Koltzenburg M, Wall PD, McMahon SB. Does the right side know what the left is doing? Trends Neurosci 1999; 22: 122-7. 